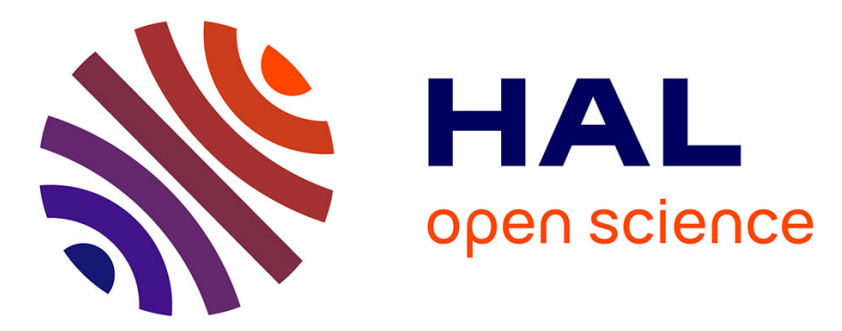

\title{
A predictive approach to jointly schedule missions and maintenances for a deteriorating vehicle
}

Elodie Robert, Christophe Bérenguer, Kéomany Bouvard, Hocéane Tedie, Romain Lesobre

\section{- To cite this version:}

Elodie Robert, Christophe Bérenguer, Kéomany Bouvard, Hocéane Tedie, Romain Lesobre. A predictive approach to jointly schedule missions and maintenances for a deteriorating vehicle. ESREL 2018 - 28th European Safety and Reliability Conference, NTNU-ESRA, Jun 2018, Trondheim, Norway. pp.529-537. hal-01887934

\section{HAL Id: hal-01887934 \\ https://hal.science/hal-01887934}

Submitted on 4 Oct 2018

HAL is a multi-disciplinary open access archive for the deposit and dissemination of scientific research documents, whether they are published or not. The documents may come from teaching and research institutions in France or abroad, or from public or private research centers.
L'archive ouverte pluridisciplinaire HAL, est destinée au dépôt et à la diffusion de documents scientifiques de niveau recherche, publiés ou non, émanant des établissements d'enseignement et de recherche français ou étrangers, des laboratoires publics ou privés.

\section{(ㅇ)(1) $\$$}

Distributed under a Creative Commons Attribution - NonCommercial - NoDerivatives $\mid 4.0$ 


\title{
A predictive approach to jointly schedule missions and maintenances for a deteriorating vehicle
}

\author{
E. Robert \\ Univ. Grenoble Alpes, CNRS, Grenoble INP, GIPSA-lab, F-38000 Grenoble, France \\ Volvo Group Trucks Technology, 69800 Saint-Priest, France \\ C. Bérenguer \\ Univ. Grenoble Alpes, CNRS, Grenoble INP, GIPSA-lab, F-38000 Grenoble, France
}

K. Bouvard \& H. Tedie

Volvo Group Trucks Technology, 69800 Saint-Priest, France

R. Lesobre

Renault Trucks Defense, 78000 Versailles, France

\begin{abstract}
Both for the manufacturer and the user of industrial vehicles, optimizing simultaneously the maintenance and missions schedule at the fleet level becomes a necessity to improve the profitability. Lots of researches have been realized to optimize either the preventive maintenance schedule or the production planning. Integrating both activities in the same schedule has become a new hotspot. Few researches have been led to schedule both maintenance operations and missions for a fleet of systems which deteriorate over time in a comprehensive predictive approach. As a first step to reach this objective, a single vehicle is considered and we propose a method to jointly optimize its predictive maintenance and its mission planning using the remaining useful life and deterioration information. The vehicle has a set of missions to complete. The aim is to group the missions in blocks and interpose maintenance operations between these blocks. However, the vehicle deteriorates over time and each mission impacts differently the deterioration according to its severity. A stochastic process is used to model the deterioration phenomenon and its parameters are changed for each mission. To obtain the best arrangement between missions and maintenance operations, a genetic algorithm is used. A criterion to minimize the maintenance cost is defined, which takes into account preventive and corrective maintenance costs associated with the missions failure probabilities. The genetic algorithm enables to approach the optimal solution in a reasonable time and avoid considering every possible arrangement.
\end{abstract}

\section{INTRODUCTION}

Many researchers have developed methods to optimize the preventive maintenance schedule for multicomponent systems (Bouvard et al. (2011)) without considering production constraints but taking into account the system availability on a fixed time period (Lesobre 2015). From these previous achievements, a new problematic has arisen aiming at jointly scheduling production and maintenance. Two strategies to solve this problem can be identified.

The sequential strategy consists in scheduling one of the two activities, maintenance or production, and using this schedule as an unavailability additional constraint to solve the joint scheduling. Benbouzid et al. (2003) develop a sequential process to schedule production activities and maintenance in flowshop workshops. The production schedule is generated using heuristic methods. Then, by considering the production tasks order as a constraint, the periodic maintenance operations are added to the initial schedule. This period is determined to reach a trade-off between the maintenance cost and the risk of reducing the machine availability. The same procedure is applied to integrate preventive maintenance in the production scheduling for a single machine to minimize the total expected weighted completion time to do all the jobs (Cassady \& Kutanoglu 2005). The exact method, for small size problems, considers all the job sequence possibilities. Then, for each possibility, all 
the feasible preventive maintenance (PM) decisions sets are tested. The job sequence-PM decisions minimizing the objective function is the optimal solution. For larger problems, a two-steps heuristic method is developed. The first step selects the job sequence having the shortest total weighted completion time. The weigh is an importance factor for each job. The second step identifies the PM decisions minimizing the total expected weighted completion time for the jobsequence established in the first step.

The integrated strategy approach aims at scheduling simultaneously maintenance and production activities. Yalaoui et al. (2014) use a linear programming model to minimize the total production cost. The production is divided in cycles and a preventive maintenance is completed at each cycle beginning to restore the production lines capacities. Each maintenance cycle cost depends on the preventive and corrective maintenance cost. The corrective one is estimated thanks to the failure rate. This exact method is satisfactory for reduced size problem. The following research works apply heuristic methods to solve the integrated scheduling problem. Feng et al. (2016) apply a genetic algorithm to minimize the costs induced by jobs tardiness, corrective and preventive maintenance action. Ladj et al. (2016) suggest an approach hybridizing genetic algorithms and artificial immune systems. A deterministic deterioration model is considered as each job deteriorates the machine of a fixed value and it is assumed that no accidental failure occurs during the time horizon. The maintenance actions are triggered when the deterioration level oversteps a failure threshold. These approaches are singleobjective optimization methods but multi-objective methods exist. Da et al. (2016) choose to minimize both the maintenance cost rate and makespan.

A comparison between the sequential approach and a new integrated strategy to minimize the manufacturing system cost for a single-unit system has been drawn by Li et al. (2010). The maintenance operations are imperfect and an improvement factor is defined to quantify each operation effectiveness. The integrated approach uses a heuristic method to find the optimal schedule and enables to save about $12 \%$ of the manufacturing costs with respect to the sequential approach. The terms sequential or integrated only refer to the way of solving the scheduling problem. In both cases, the result is a single simultaneous schedule for production and maintenance.

In the previous contributions, the deterioration modelling considers either the machine age or a deterministic model where each job deterioration is exactly known. Li et al. (2010) do not consider the failure risk or any reliability based constraints to obtain the joint schedule.

The objective of this paper is to develop a new integrated strategy to schedule both missions and maintenance for a deteriorating vehicle using deterioration information. Firstly, the joint scheduling problem is defined. Then, the adopted approach to solve it is explained. The models for the vehicle deterioration evolution and for the missions impact on the deterioration are developed and the optimization method is exposed. Finally, performances and sensitivity studies are presented on application examples to illustrate the algorithm behaviour.

\section{PROBLEM FORMULATION}

A single vehicle has a set of missions to complete. It is modelled as a single-unit system which deteriorates over time according to its activity. The vehicle operates in missions with different severity levels, characterized not only by their duration but also by several environment parameters as road condition, topography. The vehicle usage modelling is then adapted and takes into account the mission severity level.

The objective of the present work is to jointly optimize the vehicle predictive maintenance and its mission planning using the deterioration information (Figure 1). The preventive maintenance operations have to be well planned to prevent immobilizing failures, maximize the vehicle availability and not disturb the missions progression. According to these conditions, the schedule is defined as a series of mission blocks interrupted by maintenance operations to restore the vehicle deterioration state to an as good as new (AGAN) state.

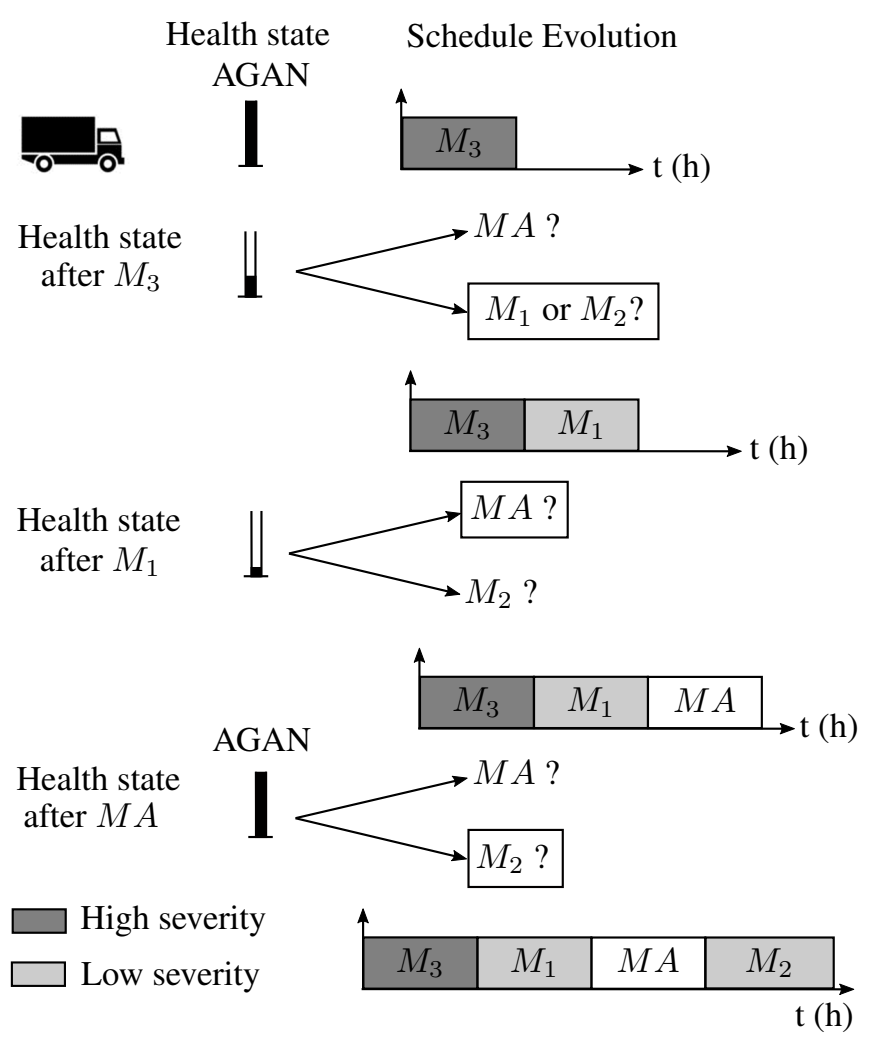

Figure 1: Framework to schedule the three missions $M_{1}, M_{2}$ and $M_{3}$ and the maintenance actions $M A$ according to the health state. Their durations and deterioration severities are respectively defined by the boxes lengths and colors.

The schedule optimization is based on the total maintenance cost and aims at maximizing the vehi- 
cle activity between two consecutive preventive maintenance operations while considering its health state. The total maintenance cost is then based on the preventive maintenance costs $C_{p t}$, for which each operation costs $C_{0}$, and the costs $C_{c t}$ associated with corrective maintenance. To estimate $C_{c t}$, the missions failure risks and the corrective maintenance cost $C_{f}$ for each operation are considered.

\section{RESOLUTION APPROACH}

\subsection{Vehicle deterioration model}

\subsubsection{Deterioration evolution}

The vehicle is characterized by a global health indicator. Its deterioration evolution is modelled by a stationary Gamma process. The choice of this stochastic continuous deterioration process is motivated by its ability to represent observable and gradual deterioration phenomena in industrial systems (Lesobre 2015, Van Noortwijk 2009). A Gamma process $X(t), t>0$ is defined by its shape and scale parameters respectively denoted $\alpha$ and $\beta$. A failure occurs when the cumulated deterioration $X(t)$ exceeds the failure threshold $L$. The distribution function $F$ followed by the time to failure $T$ is then given by Eq.1. The failure probability for a mission whose duration is equal to $t_{m}$ is equal to $F\left(t_{m}\right)$.

$F(t)=P(T \leq t)=\frac{\Gamma(\alpha t, L \beta)}{\Gamma(\alpha t)}$

where $\Gamma($.$) is the Gamma function and \Gamma(\alpha ., L \beta)$ is the incomplete Gamma function defined by $\Gamma(\alpha t, L \beta)=\int_{L \beta}^{\infty} u^{\alpha t-1} e^{-u} d u$.

\subsubsection{Missions impact on the deterioration}

The missions correspond to the deliveries the truck has to complete. They are described by their durations and they affect the vehicle deterioration evolution. Indeed, the vehicle evolves in a dynamic environment influencing its deterioration. The environment evolution results from changes in the missions characteristics during the vehicle lifetime. The deteriorationthreshold failure model used in this work allows one to integrate the mission characteristics through the modification of the deterioration parameters. It is thus assumed that these changes have a time-related impact on the deterioration process, modelled by a change in the deterioration speed. The deterioration is then modelled by a Gamma process with varying parameters. Each mission is associated with a pair of parameters.

Thanks to the deterioration model, the failure probabilities can be estimated based on the distribution function followed by the time to failure. An optimization method to jointly schedule mission and maintenance can then be developed.

\subsection{Optimization method for joint scheduling}

A genetic algorithm based approach is developed to find the optimal joint schedule for maintenance and missions minimizing the total maintenance cost. The optimization criterion definition is one of the major point to discuss.

\subsubsection{Criteria definition}

The schedule is composed of mission blocks separated by preventive maintenance operations. The optimization criterion is then based on the maintenance cost associated with the schedule. This criterion relies on two elements:

- The preventive maintenance cost $C_{0}$ corresponding to the maintenance operations scheduled at each block end.

- The corrective maintenance cost $C_{f}$ related to the failure occurring within each mission block.

An optimal balance is to be found between preventive and corrective maintenance i.e. the number of blocks and the blocks filling. To determine the possible mission number in the blocks, the deterioration process is used to estimate the failure probability for a block. This failure probability considers the parameters characterizing the missions in the block.

For $p$ missions in a block, $p$ environment changes, representing the missions severity levels, occur. Figure 2 presents an example with 3 missions and 3 environment changes. The only known information are that the deterioration level is equal to 0 at the block beginning. No information on its deterioration evolution are available when the vehicle is completing the schedule.

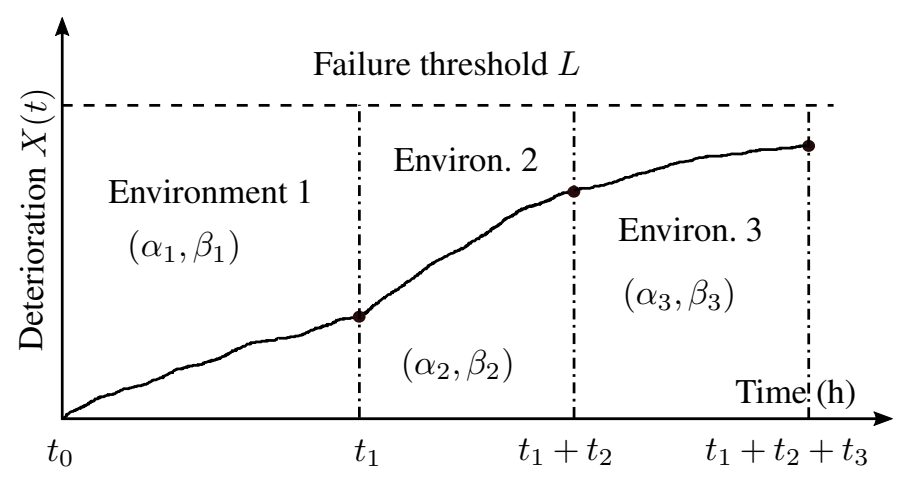

Figure 2: Deterioration evolution in a block with 3 missions

The cumulated deterioration between $t_{0}$ and $t_{1}+$ $t_{2}+t_{3}$ corresponds to the deterioration increments sum between $t_{0}$ and $t_{1}, t_{1}$ and $t_{1}+t_{2}$ and between $t_{1}+t_{2}$ and $t_{1}+t_{2}+t_{3}$. The increments probability densities of the missions are respectively denoted $f_{1}, f_{2}$ and $f_{3}$. The increments deterioration density for the block is the increments densities convolution for the missions in the block. The more numerous the environment changes are, the more complex the reliability expression is to compute (Khoury 2012). Based on 
the Gamma process properties and the environment changes, an approximation for the block deterioration process can be given by an equivalent Gamma process $G a\left(\alpha_{e}, \beta_{e}\right)$. Its average value and variance are respectively weighted mean values of the average values and variances related to the Gamma processes of the missions. The different weights are the proportions of the block duration spent in each environment. For Figure 2 , the equivalent Gamma process parameters are defined as follows:

$\frac{\alpha_{e}}{\beta_{e}}=\frac{1}{T} \sum_{i=1}^{p} t_{i} \frac{\alpha_{i}}{\beta_{i}} \quad$ and $\quad \frac{\alpha_{e}}{\beta_{e}^{2}}=\frac{1}{T} \sum_{i=1}^{p} t_{i} \frac{\alpha_{i}}{\beta_{i}^{2}}$,

where $p=3$ and $T=\sum_{j=1}^{p} t_{j}$. Note that if all the Gamma processes related to the missions have the same scale parameter $\beta$ and the same duration, the resulting convolution density is exactly the one for a Gamma process $G a\left(\sum_{i=1}^{p} \alpha_{i}, \beta\right)$.

The block failure probability is obtained based on the equivalent Gamma process. It is the probability that the cumulated deterioration exceeds the failure threshold $L$, knowing that $X\left(t_{0}\right)$, the deterioration at $t_{0}$, is equal to 0 .

$P\left(X(T)-X\left(t_{0}\right) \geq L\right)=\frac{\Gamma\left(\alpha_{e}\left(T-t_{0}\right), L \beta_{e}\right)}{\Gamma\left(\alpha_{e}\left(T-t_{0}\right)\right)}$

The criterion $C_{1}$ (Eq.4) is defined to estimate the maintenance costs for a joint schedule $\pi$ composed of $N_{b}$ blocks. It considers only one failure by block.

$C_{1}(\pi)=\sum_{k=1}^{N_{b}}\left(C_{0}+C_{f} \mathbb{P}_{f}(k)\right)$,

where $\mathbb{P}_{f}(k)$ is the probability to have one failure in the block $k$ as explained in Eq.3. Variations can then be defined to accept multi failures in the blocks. We define another criterion $C_{2}$ which considers that in a block composed of $m$ missions, no more than $m$ failures can occur. Only one failure by mission could happen. Considering multi failure in a block corresponds to estimate the expected failure occurrences in a block.

The replacement process is such that once the deterioration exceeds the failure threshold $L$, a corrective maintenance is completed and the deterioration level is back to 0. Based on the equivalent Gamma process, the deterioration evolution in the block can be characterized. The probability to have two failures in the block defined in Figure 2 can then be estimated by $P\left(X(T)-X\left(t_{0}\right) \geq 2 L\right)$. The principle is the same as the replacement process but without the deterioration level reset after a failure. The criterion $C_{2}$ for a joint schedule $\pi$ composed of $N_{b}$ blocks is:

$C_{2}(\pi)=\sum_{k=1}^{N_{b}}\left(C_{0}+C_{f} \sum_{i=1}^{N_{m}(k)} \mathbb{P}_{f_{k}}(D \geq i L)\right)$ where $N_{m}(k)$ is the number of missions in the block $k, D$ the deterioration level and $\mathbb{P}_{f_{k}}(D \geq i L)$ the probability to exceed the failure threshold $L$ for the $i^{\text {th }}$ time in the block $k$.

The two criteria $C_{1}, C_{2}$ are used as optimization criteria for the genetic algorithm developed to solve the joint scheduling problem.

\subsubsection{A genetic algorithm based method}

A genetic algorithm (GA) is developed to solve the joint scheduling problem for missions and maintenance using a maintenance cost based criterion defined in the section 3.2.1. The choice of this heuristic method is justified by its adaptability regarding the individuals and operators definitions as well as its performances to reach a good solution in a satisfying computation time. The genetic algorithm principle is based on the hybrid genetic-immune algorithm proposed by Ladj et al. (2016). The operators are adapted to be applied in a random deterioration case as Ladj et al. (2016) develop it for a deterministic deterioration case. Its general main principle is illustrated in Figure 3. The different stages are explained in the following paragraphs.

A parameter is defined to condition the block filling. As the deterioration evolution is stochastic, the exact deterioration level reached at a mission block end cannot be known. However, a failure probability can be estimated according to the missions in the block. This parameter acts on the maximum admissible failure probability for a block. It is more likely to dispatch a vehicle on a mission block whose failure probability is not too high to avoid at best corrective maintenance. This parameter is denoted $\mathbb{P}_{f_{\text {max }}}$ and its values is between 0 and 1 . The closer to 1 it is, the more flexible the genetic algorithm is to generate individuals. Acting on this parameter guides the genetic algorithm towards the best joint schedule.

Individual representation: The individuals correspond to candidate schedules for both maintenance and missions. In the GA, the genotype is obtained by sequencing the mission set into different blocks. In a block, the missions are supposed to be completed one after the other. The preventive maintenance operations occurs at each block end. For instance, in case of a problem with $n=6$ missions to schedule, a possible candidate schedule is $\pi=\{(6,2)(5,3,4)(1)\}$.

Initial population: The initial population is composed of $N_{\text {pop }}$ individuals. $60 \%$ of them are randomly generated. Each mission is randomly put in a block while respecting the block filling condition. For the remaining individuals generation, special techniques are applied. $20 \%$ of the population is generated using the First Fit (FF) method (Coffman et al. (1996)). This method is applied on random missions sequences to form candidate solutions by assigning the missions to the blocks. The last $20 \%$ is generated using to heuristic methods called First Fit Decreasing (FFD) and 
Best Fit Decreasing (BFD) (Coffman et al. (1996)). Then, block permutations are performed on these solutions to generate other individuals. Note that the individuals generated by these techniques still respect the block filling condition.

Evaluation: The evaluation stage consists in applying the fitness function Fit to evaluate the quality of an individual in the population. The fitness function is related to the maintenance cost based criteria defined in section 3.2.1. Two fitness functions for a individual $\pi$ are defined in Eq.6. The best individuals are the ones for which the maintenance costs are the lowest.

$$
F_{i t}(\pi)=\frac{1}{C_{1}(\pi)} \quad \text { and } \quad F_{i t}(\pi)=\frac{1}{C_{2}(\pi)}
$$

Selection: The selection operator is a 2-tournament selection operator (Michalewicz 1996). It randomly chooses two individuals in the current population and selects the fittest one. When the selected individuals number reaches $N_{\text {pop }}$, the selection stage is over.

Crossover: The crossover operator crosses two parent individuals to obtain new offspring individuals. A crossover probability $P_{\text {cross }}$ defines whether or not the parents will procreate. The principle is to randomly select a pair of parents. Both parent blocks are listed and a random selection is done to copy nonoverlapping blocks to both offspring individuals so that no missions are duplicated. Then, for each offspring, the remaining missions are randomly added to blocks, either an existing one while respecting the block filling condition or a new one. This principle is inspired from the crossover operator developed by Rohlfshagen and Bullinaria (2010).

Mutation: The mutation operator is based on Swap mutation (Michalewicz 1996) and consists in exchanging two randomly selected missions from two different blocks while respecting the block filling condition. This swapping occurs with a probability $P_{m u t}$.

Population dispersion: This stage periodically evaluates the total population dispersion which is of great importance to avoid converging towards a local optimal solution. The total population includes the parents and their children with their possible mutations and contains $2 N_{\text {pop }}$ individuals. As explained by Ladj et al. (2016), diversification and intensification are two major issues when building effective search algorithms. Diversification refers to the capacity to explore different regions of the search space while intensification refers to the ability to generate high fitted solutions in those regions. A balance between these two notions has to be obtained. When the iteration number $n_{i}$ is a multiple of the iteration period $i_{p}$, the population dispersion is estimated through the coefficient of variation (CV), also known as the Relative Standard Deviation (RSD) (Ladj et al. (2016)). CV considers the fitness value of each schedule $\pi_{i}$ in the total population Pop (Eq.9). According to its value, different decisions are adopted to enhance either the diversification or the intensification.

$$
C V=\frac{\sigma}{\mu} \times 100 \%
$$

with $\quad \mu=\frac{1}{2 N_{\text {pop }}} \sum_{\pi_{i} \in P o p} \frac{1}{F i t\left(\pi_{i}\right)}$

$$
\text { and } \sigma=\sqrt{\frac{1}{2 N_{p o p}} \sum_{\pi_{i} \in P o p} \frac{1}{\left(F i t\left(\pi_{i}\right)-\mu\right)^{2}}}
$$

Receptor Editing: When the population dispersion $C V$ is lower than a minimum dispersion threshold $\varepsilon_{\text {min }}$, individuals are very similar and focused on a limited search space region. The receptor editing operator aims at reducing the risk of premature convergence (Ladj et al. (2016)). A part of the least fitted individuals ( $\alpha \%$ of the population) are eliminated and replaced by random new individuals to possibly explore new search space regions.

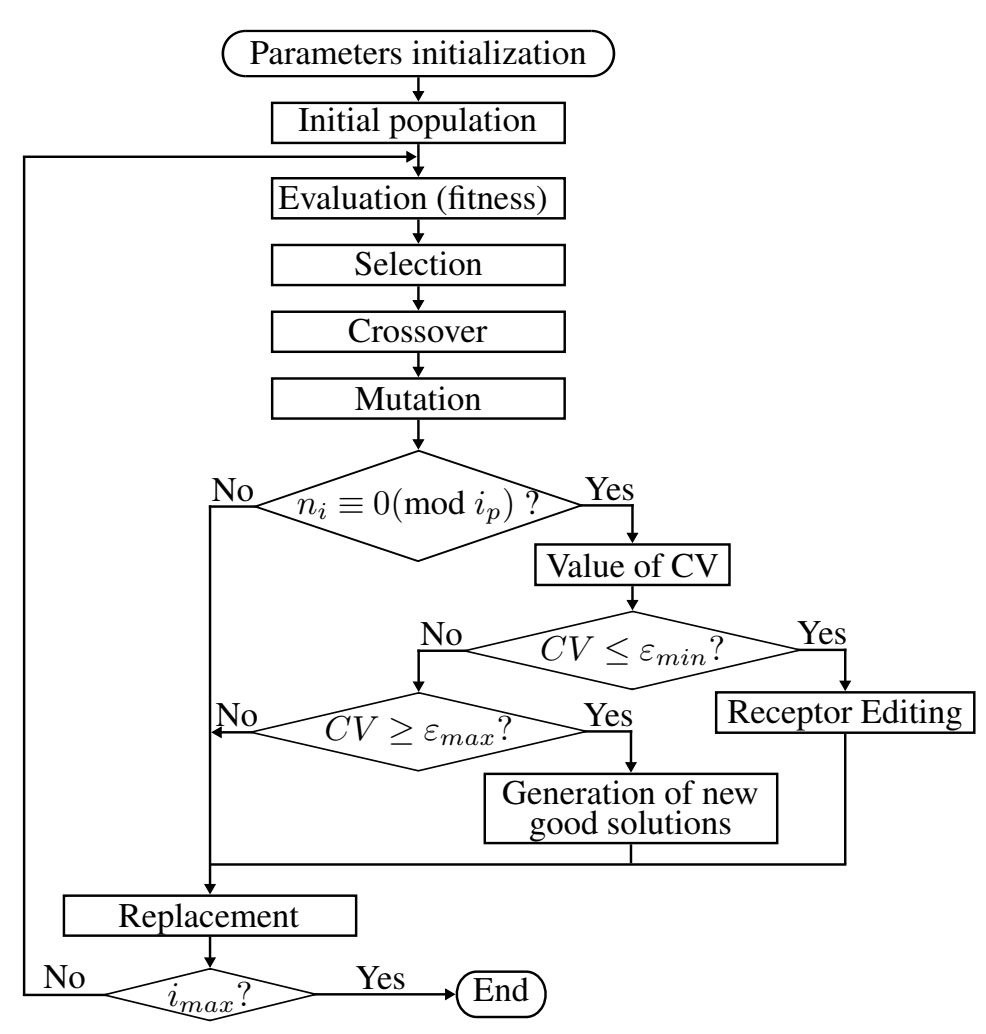

Figure 3: Genetic algorithm principle

Generation of new good solutions: When the population dispersion $C V$ is higher than a maximum dispersion threshold $\varepsilon_{\max }$, individuals cover distinct search space regions. To promote the most promising regions, a part of the least fitted individuals are replaced by mutations of the most fitted individuals ( $\alpha \%$ of the population).

Replacement: Individuals for the next generation are selected among the total population formed by the parents and the children. A part of the least fitted individuals $(\beta \%)$ is directly added to the next generation while the fittest members among parents and children 
complete the next generation until it contains $N_{\text {pop }}$ individuals.

Termination condition: The genetic algorithm terminates after $i_{\max }$ iterations and a joint schedule for missions and maintenance operations minimizing the corrective and preventive maintenance costs can be obtained. The results are illustrated through application examples.

\section{APPLICATION EXAMPLES}

\subsection{Simulated data}

Two datasets of $n=6$ missions represent two scenarii (Tables $1 \& 2$ ). The difference between them comes from the mission failure probabilities range. For the dataset $\mathrm{A}$, the maximum mission failure probability is $1 \%$ while for the dataset $\mathrm{B}$, it is $19 \%$.

Table 1: Dataset A

\begin{tabular}{|c|c|c|c|c|}
\hline Missions & Durations (h) & $\alpha_{m}$ & $\beta_{m}$ & Failure probabilities \\
\hline 1 & 21 & 0.13 & 0.1 & 0.002 \\
2 & 21 & 0.18 & 0.1 & 0.009 \\
3 & 8 & 0.4 & 0.1 & 0.004 \\
4 & 8 & 0.33 & 0.1 & 0.002 \\
5 & 2 & 1.33 & 0.1 & 0.002 \\
6 & 3 & 1.32 & 0.1 & 0.01 \\
\hline
\end{tabular}

Table 2: Dataset B

\begin{tabular}{|c|c|c|c|c|}
\hline Missions & Durations (h) & $\alpha_{m}$ & $\beta_{m}$ & Failure probabilities \\
\hline 1 & 9 & 0.85 & 0.1 & 0.189 \\
2 & 4 & 1.85 & 0.1 & 0.163 \\
3 & 3 & 1.34 & 0.1 & 0.011 \\
4 & 6 & 0.93 & 0.1 & 0.048 \\
5 & 6 & 0.90 & 0.1 & 0.041 \\
6 & 2 & 3.81 & 0.1 & 0.184 \\
\hline
\end{tabular}

The dataset A further emphasizes the influence of the missions variances changes while the dataset $\mathrm{B}$ better illustrates the sensitivity studies for the impact of the ratio between the preventive and corrective maintenance costs and for the effect of $\mathbb{P}_{f_{\max }}$.

The preventive and corrective maintenance costs $C_{0}$ and $C_{f}$, the failure threshold $L$ and the genetic algorithm parameters have to be initialized (Table 3 ). The maximum failure probability $\mathbb{P}_{f_{\max }}$ is fixed at 0.95 for all the studies except for the one on the variance changes (4.3.3) for which $\mathbb{P}_{f_{\max }}$ is equal to 0.1 .

Table 3: Parameters definition

\begin{tabular}{|c|c||c|c|}
\hline Parameter & Value & Parameter & Value \\
\hline$C_{0}$ & 1000 & $C_{f}$ & 3000 \\
$L$ & $100 \%$ & $N_{\text {pop }}$ & 30 \\
$P_{\text {cross }}$ & 0.7 & $P_{\text {mut }}$ & 0.1 \\
$i_{p}$ & 4 & $i_{\max }$ & 100 \\
$\alpha$ & $20 \%$ & $\beta$ & $20 \%$ \\
$\varepsilon_{\min }$ & 10 & $\varepsilon_{\max }$ & 60 \\
\hline
\end{tabular}

Table 4: Performance results

\begin{tabular}{|c|c|c||c|c|}
\hline & \multicolumn{2}{|c||}{ Dataset A } & \multicolumn{2}{c|}{ Dataset B } \\
\hline Criterion & $C_{1}$ & $C_{2}$ & $C_{1}$ & $C_{2}$ \\
\hline$C_{m}$ & 3787.2 & 3788.3 & 7898.4 & 7905 \\
\hline GA: $T_{c}(\mathrm{~s})$ & 2.34 & 2.90 & 3.02 & 3.24 \\
\hline EM: $T_{c}(\mathrm{~s})$ & 7.79 & 7.76 & 3.47 & 3.48 \\
\hline
\end{tabular}

\subsection{Performances analysis}

The performance analysis is realized for both datasets and both criteria defined in section 3.2.1. It is performed on a computer with Intel ${ }^{\circledR}$ Xeon $^{\circledR}$ CPU E31240 v5@3.50GHz and 16.0GB RAM. For each dataset, 1000 realizations are generated to compare the maintenance cost and computation time results between the genetic algorithm (GA) and an exact method (EM). The exact method considers all the possible schedules respecting the block filling condition and their associated criterion values to find the schedule minimizing the criterion. The maintenance cost and the computation time are respectively denoted $C_{m}$ and $T_{c}$. The study results are explained in Table 4.

Dataset A: The optimal joint schedule obtained with the exact method is the same for both criteria. This schedule is $\pi_{\text {opt }}^{12}=\{(1,3)(2,5)(4,6)\}$. The computation time gains with the genetic algorithm are quite significant: $70 \%$ for the criterion $C_{1}$ and $62.6 \%$ for the criterion $C_{2}$.

Dataset B: The optimal joint schedule obtained with the exact method differs according to the chosen criterion. For the criterion $C_{1}$, the optimal schedule is $\pi_{\text {opt }_{2}}^{1}=\{(1)(2)(4)(6)(3,5)\}$ while for the criterion $C_{2}$, it is $\pi_{\text {opt }_{2}}^{2}=\{(1)(2)(3)(4)(5)(6)\}$. The computation time gains are lower than with the dataset A. They are respectively of $13.1 \%$ with $C_{1}$ and $7 \%$ with $C_{2}$. This difference comes from the number of feasible schedules when respecting the block filling condition. Indeed, the feasible schedule number for the datasets A and B are respectively 199 and 80. It explains why the exact method is faster for the dataset $\mathrm{B}$.

For all the 1000 realizations with both datasets and criteria, the genetic algorithm converges towards the same schedule as the one obtained with the exact method. The only differences that can occur are permutations between the blocks or permutations of missions in the same block. As the criteria are computed based on the equivalent deterioration process for each block, the mission order does not have an influence on the block failure probabilities. The maintenance costs for the criterion $C_{2}$ are slightly higher because multi failures are considered in the blocks. It increases the associated corrective maintenance cost.

The genetic algorithm computation time for the criterion $C_{2}$ has increased with respect to the computation time for the criterion $C_{1}$. For the datasets $\mathrm{A}$ and $\mathrm{B}$, the increases are about $24 \%$ and $7.4 \%$. As the criterion $C_{2}$ considers multi failures probabilities, there are more calculations to do when computing the fitness values for the candidate schedules.

The results show the interest of using a genetic 
algorithm based method instead of an exact method to converge towards an optimal joint schedule for missions and maintenance while saving computation time. After showing the genetic algorithm performances, it is essential to study its sensitivity to several parameters to analyse its behaviour.

\subsection{Sensitivity study}

This section studies the influence of some parameters on the genetic algorithm behaviour, on the obtained joint schedule and on its associated performance. For each study, the dataset is selected to illustrate at best its behaviour.

\subsubsection{Impact of the ratio $R_{c}$}

The first study is interested in the impact of $R_{c}$, the ratio between the preventive and corrective maintenance costs respectively denoted $C_{0}$ and $C_{f}$. It is realized with the dataset B. $R_{c}$ varies between 0.1 and 1 with a step fixed at 0.1 and the value for the preventive maintenance cost $C_{0}$ is the one defined in Table 3.

Table 5: Optimal schedule when $R_{c}$ varies (Criterion $C_{1}$ )

\begin{tabular}{|c|c|c|}
\hline$R_{c}$ & Maintenance cost & Optimal schedule \\
\hline 0.1 & 12350 & $\{(2)(6)(4)(1)(5)(3)\}$ \\
0.2 & 9175 & $\{(6)(2)(3)(1)(4)(5)\}$ \\
0.3 & 8116.7 & $\{(4)(3)(5)(2)(1)(6)\}$ \\
0.4 & 7415.4 & $\{(4)(1)(6)(5,3)(2)\}$ \\
0.5 & 6901.5 & $\{(2)(1)(6)(5,4,3)\}$ \\
0.6 & 6345.4 & $\{(2)(6,1)(3,5,4)\}$ \\
0.7 & 5867.4 & $\{(5,3,4)(1,6)(2)\}$ \\
0.8 & 5509 & $\{(1,6)(5,4,3)(2)\}$ \\
0.9 & 5230.2 & $\{(1,6)(2)(5,3,4)\}$ \\
1 & 5007.2 & $\{(5,3,4)(2)(1,6)\}$ \\
\hline
\end{tabular}

For both criteria, the block number in the schedule decreases when $R_{c}$ decreases, i.e. when the corrective maintenance cost decreases (Table 5). When the corrective cost is very high with respect to the preventive one, the best schedule favours a high block number because grouping missions increases the failure risk in each block and leads to a significant maintenance cost inflation. On the contrary, when the two maintenance costs are quite similar, grouping the missions in few blocks is less expensive with respect to the maintenance costs.

Note that the reduction of the number of blocks is faster for the criterion $C_{1}$. When $R_{c}$ is equal to 0.5 , the optimal schedule for the criterion $C_{1}$ is composed of 4 blocks while the one for the criterion $C_{2}$ has 5 blocks.

\subsubsection{Effect of the variations of $\mathbb{P}_{f_{\max }}$}

This section studies the effect of the maximum admissible failure probability for a block on the optimal schedule computation. This probability aims at guiding the genetic algorithm to converge faster towards the optimal solution. The smaller the probability is, the stricter the constraint on the block filling is.

Table 6: Optimal schedule when $\mathbb{P}_{f_{\max }}$ varies (Criterion $C_{1}$ )

\begin{tabular}{|c|c|c|}
\hline $\mathbb{P}_{f_{\max }}$ & Maintenance cost & Optimal schedule \\
\hline 0.2 & 7905 & $\{(3)(4)(5)(6)(1)(2)\}$ \\
0.3 & 7905 & $\{(6)(5)(2)(1)(4)(3)\}$ \\
$\llbracket 0.4 ; 0.9 \rrbracket$ & 7898.4 & $\{(4)(2)(1)(3,5)(6)\}$ \\
1 & 4000 & $\{(1,5,6,4,3,2)\}$ \\
\hline
\end{tabular}

This part is illustrated for the dataset $\mathrm{B}$ with the criterion $C_{1}$. Table 6 shows that when $\mathbb{P}_{f_{\max }}$ increases, the number of blocks decreases. For most of $\mathbb{P}_{f_{\max }}$ values, the optimal schedule is composed of 5 blocks. When $\mathbb{P}_{f_{\max }}$ is equal to 1 , the optimal schedule groups all the missions in the same block because it is less expensive for the maintenance.

As expected, when the block filling constraint is relaxed, the optimal schedule is composed of less blocks. Note that when $\mathbb{P}_{f_{\text {max }}}$ is equal to 1 , the optimal schedule not necessarily counts one block. For the dataset $\mathrm{A}$, it is composed of 3 blocks for both criteria.

\subsubsection{Influence of missions variance changes}

In this part, the influence of the missions variance changes are studied to evaluate the genetic algorithm behaviour. For this specific study, the block filling condition $\mathbb{P}_{f_{\max }}$ is fixed at 0.1 . It is to be sure to illustrate only the effects due to the variance changes.

The initial dataset is the dataset A. As $\mathbb{P}_{f_{\max }}$ is reduced with respect to the part 4.2 , the optimal joint schedule differs. With both criteria, it is $\pi_{o p t}=$ $\{(2)(6)(5,1)(4,3)\}$. Based on this dataset missions, three datasets are generated. The missions durations are identical but the deterioration processes parameters are modified so that the increments expected value for each mission remains the same from one dataset to another. For each mission, the variance of the deterioration increment is either increased or decreased with respect to the initial variance (Tables 7 $\& 8)$. $\mathbb{P}$ denotes the mission failure probability.

Table 7: Datasets when increasing the variance by 2 or 5

\begin{tabular}{|c|c|c|c|c|c|c|}
\hline & \multicolumn{3}{|c|}{ Variance x2 } & \multicolumn{3}{c|}{ Variance $\mathrm{x}$} \\
\hline Missions & $\alpha_{m}$ & $\beta_{m}$ & $\mathbb{P}$ & $\alpha_{m}$ & $\beta_{m}$ & $\mathbb{P}$ \\
\hline 1 & 0.07 & 0.05 & 0.02 & 0.03 & 0.02 & 0.05 \\
2 & 0.09 & 0.05 & 0.04 & 0.04 & 0.02 & 0.09 \\
3 & 0.20 & 0.05 & 0.02 & 0.08 & 0.02 & 0.07 \\
4 & 0.16 & 0.05 & 0.01 & 0.07 & 0.02 & 0.05 \\
5 & 0.66 & 0.05 & 0.01 & 0.27 & 0.02 & 0.05 \\
6 & 0.66 & 0.05 & 0.04 & 0.26 & 0.02 & 0.09 \\
\hline
\end{tabular}

When the variances are increased by a factor 2 and 5 , the optimal joint schedules are respectively $\pi_{i_{2}}=$ $\{(5,4)(6)(1)(3)(2)\}$ and $\pi_{i_{5}}=\{(5)(6)(1)(2)(4)(3)\}$. Increasing the variances increases the number of blocks in the optimal joint schedule. Indeed, increasing the variances also increases the probability to have a failure for each mission. It is then harder to group 
Table 8: Dataset when decreasing the variance by 2

\begin{tabular}{|c|c|c|c|}
\hline & \multicolumn{3}{|c|}{ Variance $/ 2$} \\
\hline Missions & $\alpha_{m}$ & $\beta_{m}$ & $\mathbb{P}$ \\
\hline 1 & 0.27 & 0.2 & $4.2 \times 10^{-5}$ \\
2 & 0.37 & 0.2 & $5.8 \times 10^{-4}$ \\
3 & 0.79 & 0.2 & $1.1 \times 10^{-4}$ \\
4 & 0.66 & 0.2 & $2.5 \times 10^{-5}$ \\
5 & 2.65 & 0.2 & $2.7 \times 10^{-5}$ \\
6 & 2.64 & 0.2 & $7.2 \times 10^{-4}$ \\
\hline
\end{tabular}

the missions into blocks. On the contrary, when the variances are reduced by a factor 2 , the optimal joint schedule is $\pi_{d_{2}}=\{(4,6)(3,1)(2,5)\}$. The number of blocks is reduced. If the variances continue to decrease, the optimal schedule remains $\pi_{d_{2}}$ because reducing more the block number lead to failure probabilities exceeding $\mathbb{P}_{f_{\text {max }}}$. The results are explained by the fact that the failure uncertainty increases when the variance increases.

\subsection{Larger size problems}

The higher the missions number to schedule is, the harder it becomes to compare the genetic algorithm results with the exact method results owing to the exact method computation time. The number of feasible schedules becomes too numerous. When considering $n=9$ missions with $\mathbb{P}_{f_{\max }}$ equal to 0.95 , the exact method needs more than 11 days to reach the optimal schedule. With the genetic algorithm, the average computation times for the criteria $C_{1}$ and $C_{2}$ are respectively $2.63 \mathrm{~s}$ and $3.77 \mathrm{~s}$. Note that when using $C_{1}$, $16 \%$ of the 1000 realizations do not reach the optimal solution and the maintenance cost deviation represents $1.45 \%$ of the optimal maintenance cost. Considering several failures by blocks gives a better maintenance cost estimation and a more coherent cost surface from one iteration to another. The convergence is then improved and when using $C_{2}$, all the schedules are identical to the optimal one.

To improve the genetic algorithm convergence, the population size $N_{\text {pop }}$ and the maximum iteration number $i_{\max }$ can be increased, but the convergence is not guaranteed. But it will be to the detriment of the computation time. Indeed, increasing the population size and the iteration number also increase the computation time.

\section{CONCLUSION}

A static method based on a genetic algorithm is proposed to schedule missions and preventive maintenance for a vehicle by optimizing a maintenance cost based criterion. Two criteria consider either one or multi failures in the blocks and include the vehicle deterioration model, evolving due to the different mission severity. Its performances and sensitivity are described through application examples. The genetic algorithm converges towards the optimal schedule in a satisfying computation time.

The obtained results are promising and offer improvement perspectives. Dynamic information, such as the deterioration level or the failure occurrences, could be integrated to update the schedule and reduce the maintenance costs even more. New missions could be added during the schedule completion. These different points will be further investigated to evolve towards a dynamic scheduling method.

\section{REFERENCES}

Benbouzid, F., Y. Bessadi, S. A. Guebli, C. Varnier, \& N. Zerhouni (2003). Résolution du problème de l'ordonnancement conjoint maintenance/production par la stratégie séquentielle. In $4 e$ Conférence Francophone de Modélisation et de SIMulation, Toulouse, France, Volume 2, pp. 627-633.

Bouvard, K., S. Artus, C. Bérenguer, \& V. Cocquempot (2011). Condition-based dynamic maintenance operations planning $\&$ grouping. Application to commercial heavy vehicles. $R e$ liab. Eng. Syst. Saf. 96(6), 601-610.

Cassady, C. R. \& E. Kutanoglu (2005). Integrating preventive maintenance planning and production scheduling for a single machine. IEEE Transactions on Reliability 54(2), 304-309.

Coffman, E., M. Garey, \& D. Johnson (1996). Approximation algorithms for bin packing: A survey. Approximation Algorithms for NP-Hard Problems, 46-93.

Da, W., H. Feng, \& E. Pan (2016). Integrated preventive maintenance and production scheduling optimization on uniform parallel machines with deterioration effect. In IEEE International Conference on Industrial Engineering and Engineering Management, pp. 951-955.

Feng, H., W. Da, \& L. Xi (2016). Joint optimization of flowshop sequence-dependent manufacturing cell scheduling and preventive maintenance. In IEEE International Conference on Industrial Engineering and Engineering Management, pp. 946-950.

Khoury, E. (2012). Modélisation de la durée de vie résiduelle et maintenance prédictive : application à des véhicules industriels [In french]. Ph. D. thesis, Université de Technologie de Troyes.

Ladj, A., F. Benbouzid-Si Tayed, \& C. Varnier (2016). An integrated prognostic based hybrid genetic-immune algorithm for scheduling jobs and predictive maintenance. In IEEE Congress on Evolutionary Computation, pp. 2083-2089.

Lesobre, R. (2015). Modélisation et optimisation de la maintenance et de la surveillance des systèmes multi-composants - Applications à la maintenance et à la conception de véhicules industriels [In french]. Ph. D. thesis, Université Grenoble Alpes.

Li, H., M. Li, Q. Liu, \& S. Li (2010). Integrated optimization research on preventive maintenance planning and production scheduling. In IEEE International Conference on Management and Service Science, pp. 1-5.

Michalewicz, Z. (1996). Genetic Algorithms + data Structures = Evolution Programs. Springer series Artificial Intelligence. Berlin ; New York: Springer.

Rohlfshagen, P. \& J. A. Bullinaria (2010). Nature inspired genetic algorithms for hard packing problems. Annals of Operations Research 179(1), 393-419.

Van Noortwijk, J. (2009). A survey of the application of gamma processes in maintenance. Reliab. Eng. Syst. Saf. 94(1), 2-21.

Yalaoui, A., K. Chaabi, \& F. Yalaoui (2014). Integrated production planning and preventive maintenance in deteriorating production systems. Information Sciences 278, 841-861. 